\title{
Towards an integrated probabilistic nowcasting system (En-INCA)
}

\author{
M. Suklitsch, A. Kann, and B. Bica \\ Central Institute for Meteorology and Geodynamics, Vienna, Austria \\ Correspondence to: M. Suklitsch (martin.suklitsch@zamg.ac.at)
}

Received: 16 January 2015 - Revised: 17 March 2015 - Accepted: 1 April 2015 - Published: 14 April 2015

\begin{abstract}
Ensemble prediction systems are becoming of more and more interest for various applications. Especially ensemble nowcasting systems are increasingly requested by different end users. In this study we introduce such an integrated probabilistic nowcasting system, En-INCA. In a case study we show the added value and increased skill of the new system and demonstrate the improved performance in comparison with a state-of-the-art LAM-EPS.
\end{abstract}

\section{Introduction}

During recent years, ensemble nowcasting systems became of vital interest for many applications. More recently, hydrological models started using such systems to run scenarios of discharge simulations and to assess the likelihood of flash floods (e.g., Silvestro et al., 2015). In operational weather forecasting, the assessment and communication of forecast uncertainty is a crucial and challenging issue. However, although specific nowcasting systems already exist for certain interests, e.g. STEPS (Bowler et al., 2006), most of them focus on precipitation only. Of course, precipitation has the reputation to be very challenging, and especially convective precipitation with its high temporal and spatial variability is an ideal parameter for probabilistic forecasting. However, also other parameters are of equal importance if viewed in the right context. Temperature, for example, can be of utter relevance in agriculture (think about frost probabilities). Strong wind, not only related to convective weather, is a high impact parameter for civil protection agencies and forestry. A combination of relatively low wind speed, high relative humidity and freezing temperatures led to a major icing event in northern parts of Austria in late 2014. In the present study, we therefore introduce an ensemble nowcasting system which covers precipitation, temperature, humidity and wind. A case study and a long-term evaluation compared to a state-of-theart LAM-EPS is presented, rounded up by final conclusions.

\section{The En-INCA system}

The Ensemble Nowcasting System (En-INCA in short) is developed at the Central Institute for Meteorology and Geodynamics (ZAMG). It basically combines the high resolution deterministic nowcasting approach of INCA (Integrated Nowcasting through Comprehensive Analysis; Haiden et al., 2011) with the probability information provided by the ALADIN-LAEF ensemble system (Wang et al., 2011).

The INCA system, which has been under constant development for a decade, is based on blending observations and numerical weather prediction (NWP) model fields. In the current version, the (deterministic) INCA uses the NWP model ALARO (Gerard et al., 2009) as background information. It also exploits remote sensing information (such as radar and satellite data) as well as high resolution time invariant information like topography and surface type. The INCA system provides frequently updated analyses and forecasts in the nowcasting range for a domain covering Austria and its surroundings (cf. Fig. 1) at a spatial resolution of $1 \mathrm{~km}$ by $1 \mathrm{~km}$. The timing of blending between the pure observation based nowcasting and the pure NWP forecast differs from one parameter to the other and is one topic of ongoing research. For example, INCA temperature forecasts are pure (downscaled) NWP data from 6 hours into the forecast, precipitation nowcasts blend into the NWP model much earlier (about $2 \mathrm{~h}$ into the forecast). But the INCA system is also able to improve the pure NWP forecasts up to $+48 \mathrm{~h}$ using topographical downscaling and error correction (through a rather 


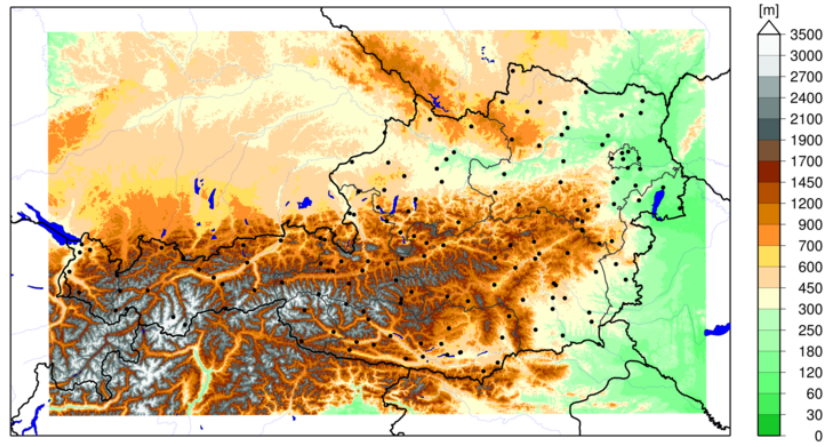

Figure 1. Operational INCA domain. Stations used in the evaluation are marked as black dots.

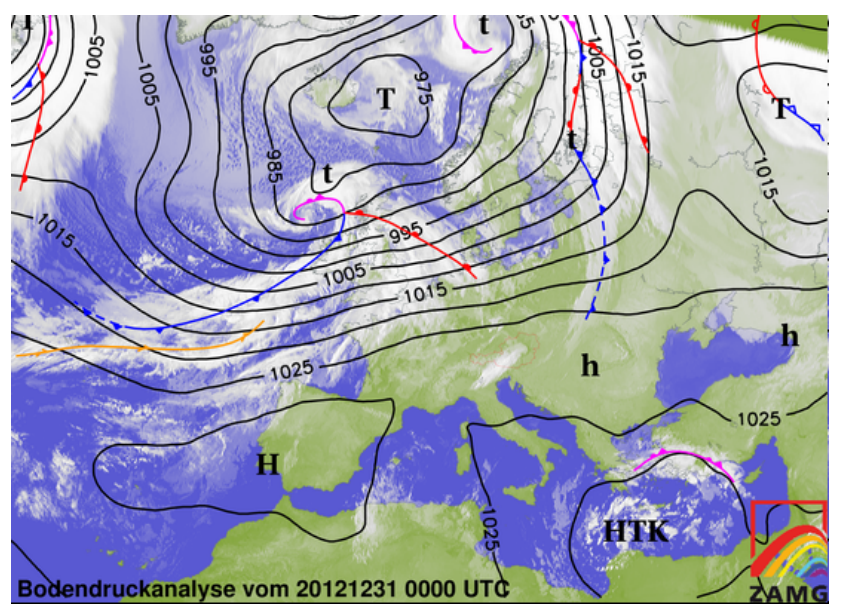

Figure 2. Frontal analysis chart with mean sea level pressure and satellite imagery for Europe on 31 December 2012, 00:00 UTC.

basic bias correction algorithm). The atmospheric fields processed with INCA include temperature, humidity (both with a vertical resolution of $200 \mathrm{~m}$ ) and wind components (vertical resolution: $125 \mathrm{~m}$ ). Surface and near surface fields provided by INCA contain wind speed and gusts, precipitation amount and type, total cloud cover and global radiation.

The second component of En-INCA is ALADIN-LAEF, the limited area ensemble forecasting system developed at ZAMG in cooperation with LACE members. The latest ensemble specifications are summarized in Table 1, the overall method is described in detail in Wang et al. (2011), and some of its components in Wang et al. (2010, 2014). A comprehensive validation of LAEF compared to a global ensemble prediction system is given by Wang et al. (2012). For En-INCA, the INCA blending algorithm is applied to all 17 members of the LAEF system individually. This yields a variety of nowcasts that combines the best of both systems: on the one hand the observation based nowcasting at very high resolution, on the other hand the probabilistic short range forecasting of a state-of-the-art LAM-EPS.
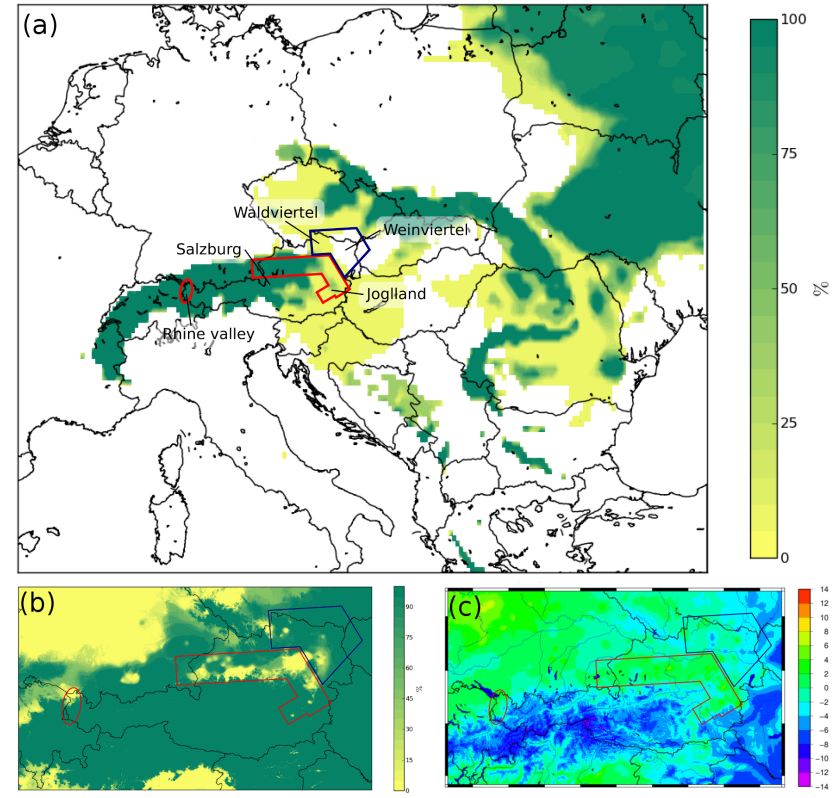

Figure 3. Probability for temperature below $0{ }^{\circ} \mathrm{C}$ as forecast by LAEF (a) and En-INCA (b) for 31 December 2012, 05:00 UTC, and the INCA temperature analysis for the corresponding time period (c). Marked regions are discussed in more detail in the text.

\section{Case study and validation}

In this section we showcase a weather situation with widespread frost on 31 December 2012. An estimate of the frost probability is of vital importance for many end users such as road maintenance services. The ZAMG analysis chart for 00:00 UTC of that day (cf. Fig. 2) shows a weak eastbound cold front that had crossed the Alps the day before. A high pressure area stretches from the Iberian Peninsula to the Alpine region and to the Balkans and subsequently gains significance for the weather in Austria. This leads to a situation favorable for strong nighttime cooling in winter: a weak pressure gradient and a cool air mass.

Figure $3 \mathrm{a}$ shows the frost probability for the morning of 31 December as forecast by LAEF $17 \mathrm{~h}$ in advance. Focusing on the regions Waldviertel and Weinviertel in northeastern Austria one can make out relatively low probabilities for temperatures below the freezing level. High frost probabilities are forecast only for the main Alpine ridge and the northern Prealps between Baden-Württemberg and Upper Austria. Figure $3 \mathrm{~b}$ shows the $5 \mathrm{~h}$ En-INCA forecast for the same validity period. The striking spatial structure of the probability distribution is a result of the higher spatial resolution of the model and the topographic downscaling. Focusing on regional and local patterns one can see considerable differences in frost probabilities, especially in the southern and eastern low lying areas of Austria, in the Waldviertel as well as in the far West of Austria (marked with colored polygons). 
Table 1. Key specifications of the ALADIN Limited Area Ensemble Forecasting (LAEF) system.

\begin{tabular}{ll}
\hline criterion & specification \\
\hline spatial resolution & horizontal: $10.9 \mathrm{~km}$ grid spacing, vertical: 45 levels \\
ensemble size & 17 members (1 control, 16 perturbed) \\
forecast range & $+72 \mathrm{~h}$ \\
perturbation model & revised multiphysics scheme (ALARO/ALADIN physics); Wang et al. (2011) \\
model surface perturbation & ensemble CANARI (Code for the Analysis Necessary for ARPEGE for its Rejects \\
& and its Initialisation) with perturbed observations \\
upper air perturbation & breeding-blending cycle (blending of small scale perturbations from ALADIN bred \\
& vectors into ECMWF-EPS initial conditions; Wang et al., 2014) \\
\hline
\end{tabular}

In order to show the added value of En-INCA during the nowcasting period, Fig. 3c shows the INCA analysis of $2 \mathrm{~m}$ temperature for 31 December 2012, 05:00 UTC. For the Alpine region itself both LAEF and En-INCA forecast high frost probabilities. Since the analysis shows widespread freezing temperature also in the Alpine valleys, one can argue that both ensemble systems have delivered a good forecast there. In the area around Lake Constance and the Rhine valley southward the analysis shows temperatures slightly above the freezing point, which is also true for most parts of Germany covered by the INCA domain, as well as a narrow strip ranging from Salzburg along the northern Prealps and the Eastern tip of the Alps southward into the Joglland (marked red in all subfigures of Fig. 3). En-INCA did not catch that pattern in its entirety (it shows high probabilities in the southeastern part of the frost free area as well as in the Vorarlberg Rhine valley, which considers the non-observed event of frost as being very likely). However, it outperformed LAEF - which did not show low frost probabilities in those frost free parts at all - by far. Finally, En-INCA "corrected" the initially frost free forecast for Waldviertel and Weinviertel by LAEF to a near certainty for frost (marked blue in Fig. 3). The temperature analysis shows that En-INCA was correct there as well.

This case study emphasizes that probabilistic nowcasting methods can yield an added value over coarser resolved LAEF forecasts, especially in the topographically challenging Alpine region. Frost probabilities gave better indications for the observed situation in En-INCA both in its spatial structure and its spatial extent. The scope of application comprises e.g. pomiculture and viticulture which both could profit of such short term, probabilistic temperature forecasts. Especially late frost events (as it happened, for example, in May 2011) often cause widespread frost damage and subsequently crop shortfalls. By provision of better forecasts containing event risks farmers can be warned and take preventive actions.

A more comprehensive evaluation of the performance of En-INCA as compared to LAEF was done based on one month of 36-hour forecasts of air temperature and relative humidity. The evaluation should determine if En-INCA can systematically outperform the coarser resolved LAEF system in the nowcasting range over a longer period of time. Reference values for the validation were averages of all available (about 150) Austrian TAWES stations (semi-automatic weather stations), marked with black dots in Fig. 1. Figure 4 shows various statistical measures and scores of the evaluation. The measures are described in detail in several books on statistics, e.g. Wilks (2011). In the top left panel the temperature bias of LAEF is rather constant over time at about $-0.5 \mathrm{~K}$. En-INCA can reduce the bias to virtually zero for the first $3 \mathrm{~h}$ before it subsequently converges to (and slightly exceeds) that of LAEF. In case of relative humidity (lower left panel in Fig. 4) the improvements persist through the 36-hour validation period: LAEF shows a mean bias of around $7.5 \%$, which through En-INCA is reduced to virtually zero for the first 5 hours. After that the bias converges to around $1.5 \%$ for the remainder of the validation period. Another interesting feature is the increase of spread in En-INCA for both parameters, whereas the RMSE, at least in case of temperature, does not exceed the one of LAEF. Most of this feature comes from the increased horizontal resolution of the underlying topography.

The two plots in the right column of Fig. 4 illustrate the Continuous Ranked Probability Score (CRPS) and the percentage of outliers. Ideally, both of them are as low as possible. The CRPS compares a full distribution of ensemble predictions with observations, both represented as cumulative distribution functions. For temperature, the CRPS is improved from $1.6 \mathrm{~K}$ (LAEF) to $0.75 \mathrm{~K}$ or less (En-INCA) for the first $3 \mathrm{~h}$ of the forecast, but even after one day the CRPS of En-INCA is slightly better (by $0.1 \mathrm{~K}$ ). In case of relative humidity En-INCA also has a smaller CRPS, however, from forecast hour 9 on the two CRPS curves are virtually identical. The percentage of outliers shows how many observed values lie outside the full forecasted range. For the present ensemble size of 18 members the percentage of outliers should be less than about $10 \%$ in order for the ensemble system to be called a reliable one. This number is out of reach for both systems, LAEF and En-INCA. Still, there is considerable improvement by En-INCA in both parameters even beyond the nowcasting range. In case of tempera- 

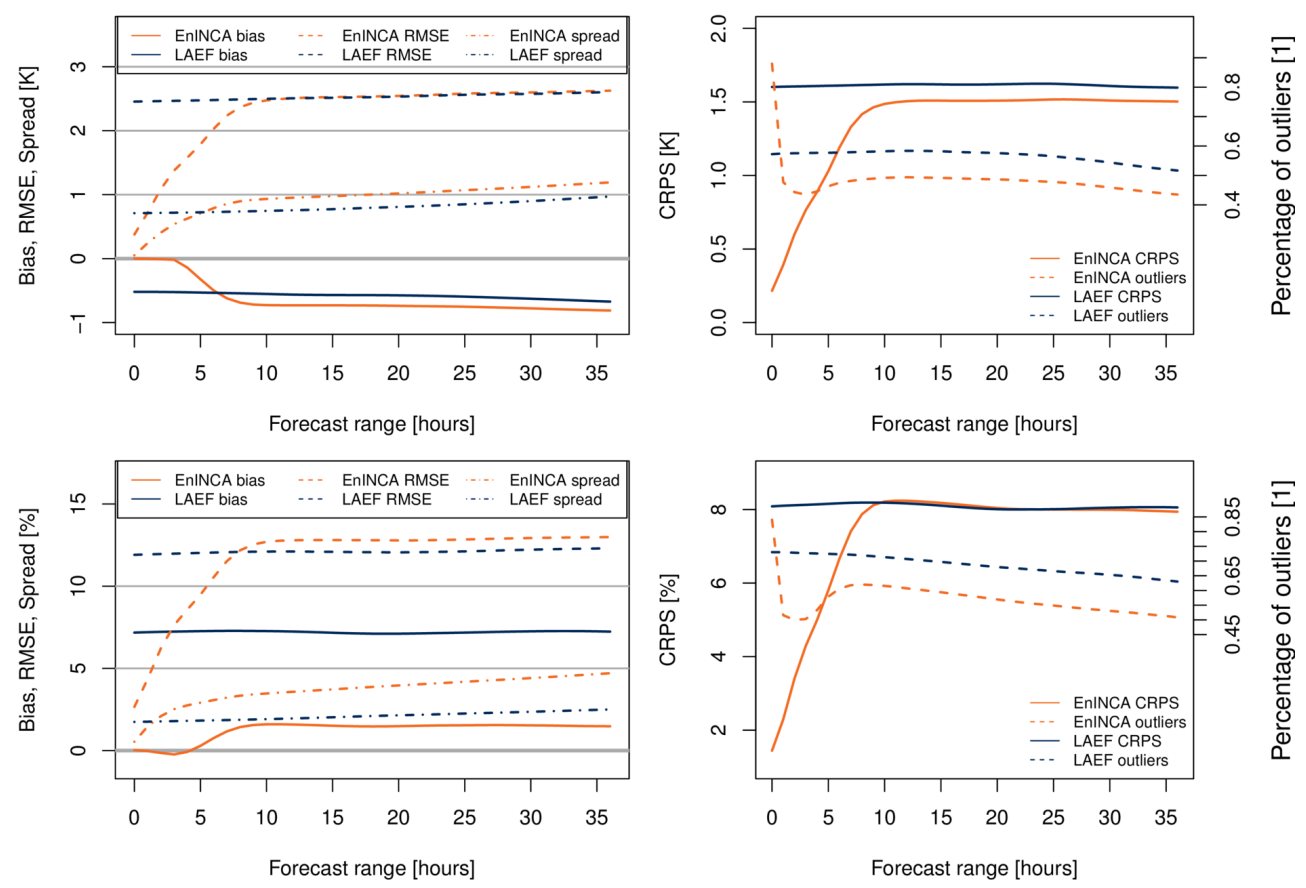

Figure 4. Various statistical measures for the one month long (January 2013) evaluation of 36-hour forecasts with En-INCA (orange) and LAEF (blue). Left panel: bias (solid lines), RMSE (dashed) and error spread (dash-dotted); Right panel: Continuous Ranked Probability Score (CRPS; solid) and percentage of outliers (dashed). Top row shows results for temperature, bottom row for relative humidity.

ture the percentage of outliers is reduced from $52 \%$ (LAEF) to $43 \%$ (En-INCA), for relative humidity it is reduced from $63 \%$ (LAEF) to $51 \%$ (En-INCA). It has to be noted, however, that the relatively large percentage of outliers of EnINCA at the beginning of the forecast is not a drawback, but rather a non-problematic feature of the system itself, since it stems from the spread close to zero.

\section{Summary}

In numerical weather prediction uncertainties exist from the very beginning of the forecast. The sources of uncertainty are numerous and widespread. By design, NWP models cannot capture all of them in their full extent. Therefore, the variability of ensemble prediction systems is often underdispersive, also during the first couple of forecast hours.

In this study we introduced an integrated probabilistic nowcasting system which at the moment covers the four parameters: temperature, relative humidity, wind and precipitation. In an orographically complex region like Austria, such systems are needed to provide end users, from civil protection authorities to private services, with spatially and temporally highly resolved probabilistic nowcasts in order to facilitate their decision making.

We showed that the mixed statistical-dynamical approach of En-INCA can improve the skill of ensemble forecasts. Apart from a better reproduction of regional features which is mainly due to the orographical downscaling, mean values over a longer period of time could be improved as well. On the basis of an exemplary validation for January 2013 we showed that the mean temperature and relative humidity bias over a set of several dozen locations could be virtually removed in the nowcasting range. Even for a longer forecasting period most statistical measures show that En-INCA outperforms LAEF.

In the presented first version of an ensemble nowcasting system only uncertainties that already exist in the LAEF system are taken into account. However, during the processing chain one can spot many elements that are affected by uncertainty. It starts in the analysis which contains errors in measurements (both of in-situ and remote sensing origin), errors in representativeness and errors made in the interpolation process. Including them should also reduce the issue of underdispersion (and thus high percentage of outliers) at forecast start. Parameters not covered by this study may be subject to even more uncertainties. For example precipitation is indirectly measured by deducing rainfall intensities from radar reflectivities. These additional sources of uncertainty are subject to future research.

Acknowledgements. The authors thank first and foremost B. Stuhl who did most of the evaluation work. We further thank F. Weidle for the provision of LAEF data and visualization, and Y. Wang for support and discussion. 
Edited by: A. Montani

Reviewed by: two anonymous referees

\section{References}

Bowler, N. E., Pierce, C. E., and Seed, A. W.: STEPS: A probabilistic precipitation forecasting scheme which merges an extrapolation nowcast with downscaled NWP, Q. J. Roy. Meteorol. Soc., 132, 2127-2155, doi:10.1256/qj.04.100, 2006.

Gerard, L., Piriou, J. M., Brozkova, R., Geleyn, J.-F., and Banciu, D.: Cloud and precipitation parameterization in a meso-gamma scale operational weather prediction model, Mon. Weather Rev., 137, 3960-3977, doi:10.1175/2009MWR2750.1, 2009.

Haiden, T., Kann, A., Wittmann, C., Pistotnik, G., Bica, B., and Gruber, C.: The Integrated Nowcasting through Comprehensive Analysis (INCA) system and its validation over the Eastern Alpine region, Weather Forecast., 26, 166-183, doi:10.1175/2010WAF2222451.1, 2011.

Silvestro, F., Rebora, N., Cummings, G., and L. Ferraris: Experiences of dealing with flash floods using an ensemble hydrological nowcasting chain: Implications of communication, accessibility, and distribution of results, J. Flood Risk Manage., doi:10.1111/jfr3.12161, in press, 2015.
Wang, Y., Kann, A., Bellus, M., Pailleux, J., and Wittmann, C.: A strategy for perturbing surface initial conditions in LAMEPS, Atmos. Sci. Lett., 11, 108-113, 2010.

Wang, Y., Bellus, M., Wittmann, C., Steinheimer, M., Weidle, F., Kann, A., Ivatek-Šahdan, S., Tian, W., Ma, X., Tascu, S., and Bazile, E.: The Central European limited-area ensemble forecasting system: ALADIN-LAEF, Q. J. Roy. Meteorol. Soc., 137, 483-502, doi:10.1002/qj.751, 2011.

Wang, Y., Tascu, S., Weidle, F., and Schmeisser, K.: Evaluation of the Added Value of Regional Ensemble Forecasts on Global Ensemble Forecasts, Weather Forecast., 27, 972-987, 2012.

Wang Y., Bellus, M., Geleyn, J.-F., Ma, X., Tian, W., and Weidle, F.: A New Method for Generating Initial Condition Perturbations in a Regional Ensemble Prediction System: Blending, Mon. Weather Rev., 142, 2043-2059, 2014.

Wilks, D.: Statistical Methods in the Atmospheric Sciences - 3rd Edn., International Geophysics, 100, ISBN 978-0-12-385022-5, 2011. 